\title{
USE OF STRUMINSKIY'S CONCEPT TO ANALYSE THE FLUCTUATING TEMPERATURE GRADIENT FIELD
}

\section{NARAYAN CHANDRA GHOSH}

Department of Mathematics, S.B. College of B.U., Mogra (W.B) 712148, India.

(Received : 27 April 1994; accepted: 24 March 1995)

Key words: Fluid dynamics, turbulent flow.

\section{INTRODUCTION}

In the study of random fluctuation of scalar and vector fields relating to turbulent state, the use of standard techniques are well known. ${ }^{1}$ Starting with the very popular Navier - Stokes equations coupled with equations of continuity valid for the fluctuating velocity components along with fluctuating pressure parts the said authors derived the tensorial equation in terms of second and third - order correlations of velocities at two points in the form of:

$$
\frac{\partial}{\partial t}\left(\overline{u_{i} u_{j}^{\prime}}\right)-\frac{\partial}{\partial \xi_{k}}\left(\overline{u_{i} u_{k} u_{j}^{\prime}}+\overline{u_{j} u_{k} u_{i}^{\prime}}\right)=2 v \nabla_{\xi}^{2} \overline{u_{i} u_{j}^{\prime}}
$$

under the assumption of homogeneity and isotropy where the terms $\overline{p u_{j}^{\prime}}=\overline{p^{\prime} u_{i}}=0$. Double and triple velocity correlations are:

$$
\overline{u_{i} u_{j}^{\prime}}=\bar{u}^{2} R_{i j}=\bar{u}^{2}\left[-\frac{1}{2 r} \frac{\partial f}{\partial r} \xi_{i} \xi_{j}+f+\frac{r}{2} \frac{\partial f}{\partial r} \delta_{i j}\right]
$$

and

$$
\overline{u_{u} u_{j}^{\prime} u_{k}}=\left(\bar{u}^{2}\right)^{3 / 2} T_{i k j}=\left(\bar{u}^{2}\right)^{3 / 2}\left[\frac{r h-h}{r^{3}} \xi_{i} \xi_{j} \xi_{k}-\frac{r \frac{\partial h}{\partial r}+2 h}{r}\left(\xi_{i} \delta_{j k}+\xi_{k} \delta_{i j}\right)+\frac{h}{r} \xi_{k} \delta_{i j}\right]
$$

The reduced scalar version of equation (1) as deduced by Karman and Howrath ${ }^{2}$ is as follows:

$$
\frac{\partial}{\partial t}\left\{\bar{u}^{2} f\right\}+2\left(\bar{u}^{2}\right)^{3 / 2}\left[\frac{\partial h}{\partial r}+\frac{4}{r} h\right]=2 v \bar{u}^{2}\left[\frac{\partial^{2} f}{\partial r^{2}}+\frac{1}{4} \frac{\partial f}{\partial r}\right]
$$


from which one can have single scalar equation in terms of two unknown scalars f and h.

Now let us start with the well-known equation for the decay of fluctuating temperature field variables $\mathrm{T}^{\prime}$ at a point $\mathrm{A}\left(\mathrm{X}^{\prime}\right)$ in a homogeneous and isotropic turbulent flow field as:

$\frac{\partial T^{\prime}}{\partial t}+\frac{\partial}{\partial X_{i}^{\prime}}\left(u_{l}^{\prime} T^{\prime}\right)=K \nabla_{x^{\prime}}^{2} T^{\prime}$

where that is viewed as a passived substance while radiative heat exchange and the heat flux related to the dissipation of kinetic energy are neglected, lth component of the fluctuating velocity at $\mathrm{A}\left(X^{\prime}\right)$ for the relevant time $t$ is represented by $u_{l}^{\prime}$ and $\mathrm{K}$ is the coefficient of thermal diffusivity of the medium under consideration.

At a neighbouring point in the same medium fluctuating temperature similarly can be written as:

$$
\frac{\partial T^{\prime \prime}}{\partial t}+\frac{\partial}{\partial X_{i}^{\prime \prime}}\left(u_{l}^{\prime \prime} T^{\prime \prime}\right)=K \nabla_{x^{\prime \prime}}^{2} T^{\prime \prime}
$$

For consideration of the fluctuating temperature field one can derive:

$$
\frac{\partial}{\partial t}\left(\frac{\partial T^{\prime}}{\partial x_{i}^{\prime}}\right)+\frac{\partial^{2}\left(u_{l}^{\prime} T^{\prime}\right)}{\partial x_{i}^{\prime} \partial x_{j}^{\prime \prime}}=K \nabla_{x^{\prime}}^{2} \frac{\partial T^{\prime}}{\partial x_{i}^{\prime}}
$$

and

$$
\frac{\partial}{\partial t}\left(\frac{\partial T^{\prime \prime}}{\partial x_{j}^{\prime}}\right)+\frac{\partial^{2}\left(u_{l}^{\prime \prime} T^{\prime \prime}\right)}{\partial x_{j}^{\prime \prime} \partial x_{i}^{\prime}}=K \nabla_{x^{\prime \prime}}^{2 \prime} \frac{\partial T^{\prime \prime}}{\partial x_{j}^{\prime \prime}}
$$

\section{Tensorial Equation Related to the Derived Field and its Simplification under the Assumptions of Homogeneity and Isotropy}

From the work of Strumniskiy ${ }^{3,4}$ and using the derivation of Basu ${ }^{5}$ it is possible to find a simplified version of tensorial equation related to derived field in case of homogeneous and isotropic turbulent fluid flow. 
From the relation (7) and (8) one can easily write

$\frac{\partial}{\partial t}\left(\overline{\left.\frac{\partial T^{\prime}}{\partial x_{i}^{\prime}} \frac{\partial T^{\prime \prime}}{\partial x_{j}^{\prime \prime}}\right)}\right)-\frac{\partial}{\partial \xi_{l}}\left[\overline{\left.\frac{\partial}{\partial x_{i}^{\prime}}\left(u_{l}^{\prime} T^{\prime}\right) \frac{\partial T^{\prime \prime}}{\partial x_{j}^{\prime \prime}}+\frac{\partial}{\partial x_{j}^{\prime \prime}}\left(u^{\prime \prime} T^{\prime \prime}\right) \frac{\partial T^{\prime}}{\partial x_{i}^{\prime}}\right]}\right]=2 K \nabla_{\xi}^{2}\left(\overline{\frac{\partial T^{\prime}}{\partial x_{i}^{\prime}}} \frac{\partial T^{\prime \prime}}{\partial x_{j}^{\prime \prime}}\right)$

Substituting in equation (9)

$\overline{\frac{\partial T^{\prime \prime}}{\partial x_{i}^{\prime \prime}} \frac{\partial T^{\prime \prime}}{\partial x_{j}^{\prime \prime}}} \equiv(\overline{\operatorname{grad} T})^{2} R_{i j}$

and

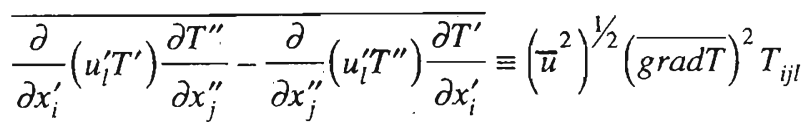

one can re-write equation (9) as follows

$\frac{\partial}{\partial t}\left[(\overline{\operatorname{grad} T})^{2} R_{i j}\right]-\frac{\partial}{\partial \xi_{l}}\left[\left(\bar{u}^{2}\right)^{1 / 2}(\overline{\operatorname{grad} T})^{2} T_{i j l}\right]=2 K \nabla_{\xi}^{2}\left[(\overline{\operatorname{grad} T})^{2} R_{i j}\right]$

with help of Robertson's ${ }^{6}$ technique we have

$(\overline{\operatorname{grad} T})^{2} R_{i j}=\frac{1}{r} \frac{\partial L}{\partial r} \xi_{i} \xi_{j}+L \delta_{i j}$

and

$$
\frac{1}{\left(\bar{u}^{2}\right)^{1 / 2}(\overline{\operatorname{grad} T})^{2}}\left(\overline{u_{l}^{\prime} T^{\prime} T^{\prime \prime}}\right)=S \xi_{l}
$$

where $\mathrm{L}$ and $\mathrm{S}$ are the scalars dependent on $(\vec{\xi} \vec{\xi})=r^{2}$ and $\mathrm{t}$.

As the case is homogeneous and isotropy reflection at the point $A$ and then $B$ may be replaced by translation of magnitude $\overrightarrow{A B}$ along $\mid \overrightarrow{A B}$. Now setting $\mid \overrightarrow{A B}$ as $\perp$ direction for $\mathrm{i}=\mathrm{j}=2$ or $\mathrm{i}=\mathrm{j}=3$ with the prescribed conversions we have with the help of $\xi_{1}=r, \xi_{2}=\xi_{3}=0$. The final scalar form can be written as 


$$
\frac{\partial}{\partial t}\left[(\overline{\operatorname{grad} T})^{2} L\right]+2\left(\bar{u}^{2}\right)^{1 / 2}(\overline{\operatorname{grad} T})^{2}\left(\frac{\partial^{2} S}{\partial r^{2}}+\frac{4}{r} \frac{\partial S}{\partial r}\right) \equiv 2 K(\overline{\operatorname{grad} T})^{2}\left(\frac{\partial^{2} L}{\partial r^{2}}+\frac{4}{r} \frac{\partial L}{\partial r}\right)
$$

\section{DISCUSSION}

Equation (12) is similar in nature to equation (1) as obtained by Karman and Howarth. ${ }^{2}$ Scalar version of (1) and (12) as given in the relations (4) and (15) respectively are also similar in nature. Alhough in case of (4) the component along $\overrightarrow{A B}$ is considered but in the derivation (15) the component perpendicular to $\mathrm{AB}$ has been taken into consideration. The direction 2 and 3 are equivalent in this context. This is due to the characteristic of a gradient field. Just like the scalar version of Karman and Howarth equation (4), the equation (15) contains two scalar functions $L$ and $S$. As $L$ and $S$ are not determinable by only one equation (15) one need frame another equation containing third order correlation.

\section{References}

1. Taylor G.K. (1955). Statistical theory of turbulence Part I - IV. Proceedings of Royal Society Series A: 151-421.

2. Karman T.V. \& Howarth L. (1938). On the statistical theory of isotropic turbulence. Proceedings of Royal Society Series A: 164-192.

3. Struminskiy V.V. (1978). A new trend in analysis of turbulence. Fluid Mechanics - Soviet Research 7(1): 23-28.

4. Struminskiy V.V. (1978). Introductory remarks at the opening of All.Union Seminar on Turbulent Flow of Liquids and Gases. Fluid Mechanics - Soviet Research 7(1): 1-2.

5. Basu M. (1982). Ph.D. dissertation, Calcutta University 28-39.

6. Robertson H.P. (1940). The invariant theory of isotropic turbulence. Proceedings of Cambridge Philosophical Society 36(2): 209-218. 